\title{
EDITORIAL
}

For reprint orders, please contact: reprints@futuremedicine.com

\section{Is low and slow the way to go? Metronomic therapy in the treatment of pediatric brain tumors}

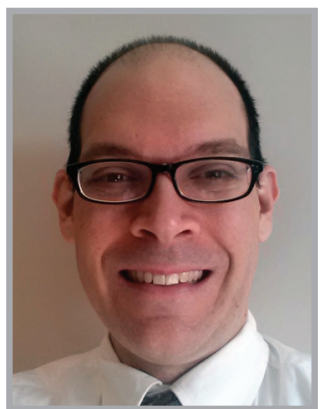

David S Wolf'

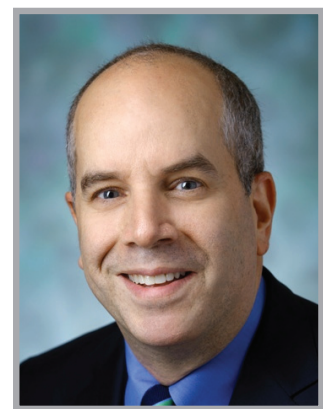

Kenneth J Cohen*2 “...low repetitive doses of chemotherapeutics ... [have]

a number of potential benefits, including better tolerability and

outpatient/home administration, coupled with less systemic toxicity and neurotoxicity."

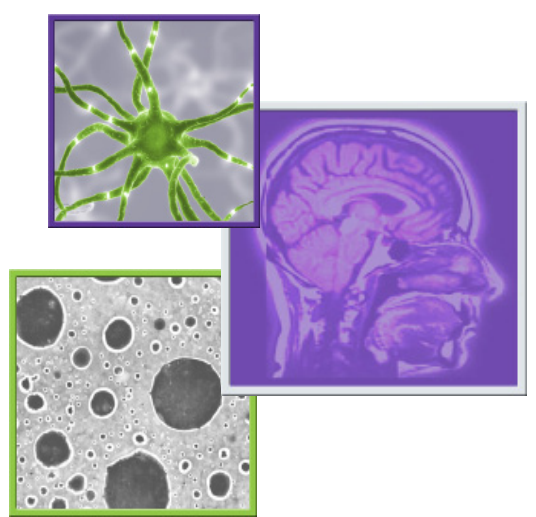

Metronomic chemotherapy refers to the use of repetitive, low-dose, often orally administered chemotherapy given frequently (often daily or weekly) without long breaks between cycles of treatment. This dosing strategy gained favor when studies in animal models demonstrated that mice with tumor xenografts could be successfully treated with low repetitive doses of chemotherapeutics, even when the tumor was resistant to the same chemotherapeutic when administered in high intermittent doses [1,2]. This strategy has a number of potential benefits, including better tolerability and outpatient/home administration, coupled with less systemic toxicity and neurotoxicity. The mechanism of action in metronomic therapy is not fully elucidated, but a primary effect is on tumor angiogenesis. Antiangiogenic therapy can inhibit neovascularization, thereby disrupting the primary tumor's blood supply, and preventing tumor progression and metastasis. Under the typical dose-intense chemotherapy regimens, there is a prolonged period between cycles of chemotherapy, offering a window of opportunity for the tumor to resume endothelial proliferation. Other mechanisms outside of antiangiogenesis have also been proposed. Enhanced anti-tumor immunity via downregulation of regulatory $\mathrm{T}$ cells has been demonstrated in vitro using chemotherapies commonly utilized in metronomic schedules [3] and in vivo in glioma models using metronomic chemotherapy [4]. Induction of tumor dormancy, the state of either cell cycle arrest, or balance of cell proliferation and apoptosis, has also been theorized as a mechanism of action [5]. One added potential mechanism of action is the ability of the metronomic chemotherapeutic(s) to saturate repair enzymes that diminish the utility of chemotherapy. This rationale is proposed for the use of low-dose temozolomide in the treatment of glioblastoma with nonmethylated (i.e., active $O^{6}$-methylguanine methyltransferase). It is believed that low-dose temozolomide overwhelms the capacity of this DNA repair enzyme [6].

Application of metronomic dosing in the clinic for children with brain tumors has demonstrated some encouraging

'Department of Neurology, The Johns Hopkins Hospital, 1800 Orleans Street, Bloomberg 11379, Baltimore, MD 21287, USA ${ }^{2}$ The Sidney Kidney Kimmel Comprehensive Cancer Center, The Johns Hopkins Hospital, 1800 Orleans Street, Bloomberg 11379, Baltimore, MD 21287, USA

*Author for correspondence: Tel.: +1 410614 5055; Fax: +1 410955 0028; kcohen@jhmi.edu

\footnotetext{
"While it seems plausible to integrate metronomic strategies into the upfront treatment of children with certain high-risk brain tumors ... it will be more challenging to thoughtfully integrate metronomic dosing into the upfront treatment of children with embryonal tumors."
} 
“...metronomic chemotherapy has been shown to offer some benefit to children with specific recurrent brain tumors." results. Metronomic dosing of topoisomerase inhibitors has demonstrated utility in multiple tumor types. Topotecan administered in 21 days out of a 28 day schedule resulted in prolonged remission in two children with disseminated primitive neuroectodermal tumors and stable disease in four out of the 26 evaluable patients [7]. Low-dose etoposide is a standard option in relapsed ependymoma. Needle and colleagues demonstrated that four out of five children responded to etoposide, with one having a complete response [8]. Others have demonstrated a response rate ranging from 50 to $83 \%$ $[9,10]$. With both chemotherapies, hematologic toxicity is the most common adverse outcome observed.

In 2005, Kieran and colleagues demonstrated that a multiagent regimen consisting of thalidomide and celecoxib given continuously in addition to low-dose etoposide and cyclophosphamide was well tolerated in a heavily pretreated group of children with relapsed solid tumors [11]. Of the 20 patients enrolled, eight were able to complete 6 months of therapy, including four children with ependymoma, one with medulloblastoma and one with low-grade glioma. Progression-free survival was extended to $>2$ years in five out of six of these subjects. Building on that study, 16 children with recurrent embryonal brain tumors (medulloblastoma, pineoblastoma, atypical teratoid rhabdoid tumors and supratentorial primitive neuroectodermal tumors) were treated with the four-drug regimen [11] plus fenofibrate, bevacizumab and intraventricular etoposide and liposomal cytarabine. Out of the 16 subjects, ten responded well to the therapy, with a median survival of 33 months [12]. The medulloblastoma subjects had an event-free survival of $100 \%$ at 6 months and $69 \%$ at 2 years. Therapy was well tolerated, and most subjects were able to stop receiving etoposide and cyclophosphamide before the end of the first year of therapy. The international COMBAT trial used a similar antiangiogenic metronomic schema, but showed a lower response rate in the embryonal tumor subgroup [13]. Overall survival at 2 years was $33 \%$ in 12 subjects.

A number of issues need to be addressed as metronomic dosing schedules and agents are utilized in the treatment of children with brain tumors. First, some thoughtful preclinical investigation is warranted to determine which agents provide the most apparent therapeutic benefit. Some studies have utilized a 'kitchen sink' approach combining numerous agents that individually have shown some antiangiogenic activity as part of the regimen. While these strategies have generally been tolerated from a toxicity perspective, largely due to nonoverlapping toxicities among the agents, whether five to eight agents are required in order to achieve the therapeutic objective is unclear. Second, where these strategies best fit in the course of disease treatment is largely untested at present. These agents have been most commonly used in the pediatric brain tumor population at the time of replase. While activity has been demonstrated, whether the risk of relapse would be modified if metronomic strategies were utilized in the upfront setting in high-grade tumors is untested, although it has been investigated in low-grade gliomas [14]. While it seems plausible to integrate metronomic strategies into the upfront treatment of children with certain high-risk brain tumors (e.g., diffuse intrinsic pontine glioma and high-grade gliomas), it will be more challenging to thoughtfully integrate metronomic dosing into the upfront treatment of children with embryonal tumors - despite the fact that it is in these populations that this strategy has been proven most efficacious in the relapse setting. Third, the potential longterm morbidities of these therapies are uncertain. The cumulative exposure of some chemotherapeutics can be quite high, which may lead to an increased risk of second malignancies. This is of particular importance when using alkylating agents, such as temozolomide or cyclophosphamide, or topoisomerase inhibitors, such as etoposide. The effect on normal growth in children may also be affected with a metronomic, antiangiogenic regimen. Finally, it is unclear whether continuous exposure of tumor cells to low-dose chemotherapy will select clones that are resistant to therapy or if it has any effect on cancer stem cells.

In conclusion, metronomic chemotherapy has been shown to offer some benefit to children with specific recurrent brain tumors. Advantages are numerous, including easy administration, low toxicity and largely outpatient administration. Further work needs to be undertaken in order to determine which agents are required for a clinically manageable regimen, when these therapies should be integrated into treatment and whether these therapies pose long-term 
toxicity risks should a cure be achieved. Early results are promising and these strategies deserve to be studied further.

\section{Financial \& competing interests disclosure}

The authors have no relevant affiliations or financial involvement with any organization or entity with a financial interest in or financial conflict with the subject matter or materials discussed in the manuscript. This includes employment, consultancies, honoraria, stock ownership or options, expert testimony, grants or patents received or pending, or royalties.

No writing assistance was utilized in the production of this manuscript.

\section{References}

1 Klement G, Baruchel S, Rak J et al. Continuous low-dose therapy with vinblastine and VEGF receptor-2 antibody induces sustained tumor regression without overt toxicity. J. Clin. Invest. 105(8), R15-R24 (2000).

2 Browder T, Butterfield CE, Kraling BM et al. Antiangiogenic scheduling of chemotherapy improves efficacy against experimental drug-resistant cancer. Cancer Res. 60 (7), 1878-1886 (2000).

3 Tanaka H, Matsushima H, Mizumoto N, Takashima A. Classification of chemotherapeutic agents based on their differential in vitro effects on dendritic cells. Cancer Res. 69(17), 6978-6986 (2009).

4 Banissi C, Ghiringhelli F, Chen L, Carpentier AF. Treg depletion with a low-dose metronomic temozolomide regimen in a rat glioma model. Cancer Immunol. Immunother. 58(10), 1627-1634 (2009).

5 Pasquier E, Kavallaris M, Andre N. Metronomic chemotherapy: new rationale for new directions. Nat. Rev. Clin. Oncol. 7(8), 455-465 (2010).

6 Tolcher AW, Gerson SL, Denis L et al. Marked inactivation of O6-alkylguanineDNA alkyltransferase activity with protracted temozolomide schedules. Br. J. Cancer 88(7), 1004-1011 (2003).

7 Minturn JE, Janss AJ, Fisher PG et al. A Phase II study of metronomic oral topotecan for recurrent childhood brain tumors. Pediatr. Blood Cancer 56(1), 39-44 (2011).

8 Needle MN, Molloy PT, Geyer JR et al. Phase II study of daily oral etoposide in children with recurrent brain tumors and other solid tumors. Med. Pediatr. Oncol. 29(1), 28-32 (1997).

9 Sandri A, Massimino M, Mastrodicasa L et al. Treatment with oral etoposide for childhood recurrent ependymomas. J. Pediatr. Hematol. Oncol. 27(9), 486-490 (2005).

10 Chamberlain MC. Recurrent intracranial ependymoma in children: salvage therapy with oral etoposide. Pediatr. Neurol. 24(2), 117-121 (2001).

11 Kieran MW, Turner CD, Rubin JB et al. A feasibility trial of antiangiogenic (metronomic) chemotherapy in pediatric patients with recurrent or progressive cancer. J. Pediatr. Hematol. Oncol. 27(11), 573-581 (2005).

12 Peyrl A, Chocholous M, Kieran MW et al. Antiangiogenic metronomic therapy for children with recurrent embryonal brain tumors. Pediatr. Blood Cancer 59 (3), 511-517 (2012).

13 Zapletalova D, Andre N, Deak L et al. Metronomic chemotherapy with the COMBAT regimen in advanced pediatric malignancies: a multicenter experience. Oncology 82(5), 249-260 (2012).

14 Lashkari HP, Saso S, Moreno L, Athanasiou $\mathrm{T}$, Zacharoulis S. Using different schedules of Temozolomide to treat low grade gliomas: systematic review of their efficacy and toxicity. J. Neurooncol. 105(2), 135-147 (2011). 